\title{
Branching processes in random environment die slowly
}

\author{
Vladimir A. Vatutin 1 and Andreas E. Kyprianou非 \\ ${ }^{1}$ Steklov Mathematical Institute RAS, Gubkin street 8, 19991, Moscow, Russia \\ ${ }^{2}$ The University of Bath, Claverton Down, Bath, BA2 7AY, UK
}

Let $Z_{n}, n=0,1, \ldots$, be a branching process evolving in the random environment generated by a sequence of iid generating functions $f_{0}(s), f_{1}(s), \ldots$, and let $S_{0}=0, S_{k}=X_{1}+\ldots+X_{k}, k \geq 1$, be the associated random walk with $X_{i}=\log f_{i-1}^{\prime}(1), \tau(m, n)$ be the left-most point of minimum of $\left\{S_{k}, k \geq 0\right\}$ on the interval $[m, n]$, and $T=\min \left\{k: Z_{k}=0\right\}$. Assuming that the associated random walk satisfies the Doney condition $P\left(S_{n}>0\right) \rightarrow \rho \in(0,1), n \rightarrow \infty$, we prove (under the quenched approach) conditional limit theorems, as $n \rightarrow \infty$, for the distribution of $Z_{n t}, Z_{\tau(0, n t)}$, and $Z_{\tau(n t, n)}, t \in(0,1)$, given $T=n$. It is shown that the form of the limit distributions essentially depends on the location of $\tau(0, n)$ with respect to the point $n t$.

Keywords: critical branching process, random environment, limit theorems

\section{Introduction}

Recently a number of papers appeared (see, for instance, (1), (10), (12)-(19)) dealing with branching processes in random environment in which individuals reproduce independently of each other according to random offspring distributions which vary from one generation to the other. The present article complements results established in (13)-(19) where critical branching processes in random environment were investigated under the quenched approach. To give a formal description of the model under consideration we shall spend some time in this section introducing notation before proceeding to the main results in the next section.

Let $\Delta$ be the space of probability measures on $\mathbb{N}_{0}:=\{0,1,2, \ldots\}$. Equipped with the metric of total variation $\Delta$ becomes a Polish space. Let $\mathbf{K}$ be a random variable taking values in $\Delta$. An infinite sequence $\overline{\mathbf{K}}=\left(\mathbf{K}_{0}, \mathbf{K}_{1}, \ldots\right)$ of i.i.d. copies of $\mathbf{K}$ is said to form a random environment. A sequence of $\mathbb{N}_{0^{-}}$ valued random variables $Z_{0}, Z_{1}, \ldots$ is called a branching process in the random environment $\overline{\mathbf{K}}$, if $Z_{0}$ is independent of $\overline{\mathbf{K}}$ and given $\overline{\mathbf{K}}$ the process $Z=\left(Z_{0}, Z_{1}, \ldots\right)$ is a Markov chain with

$$
\mathcal{L}\left(Z_{n+1} \mid Z_{n}=z(n), \overline{\mathbf{K}}=\left(\mathbf{k}_{0}, \mathbf{k}_{1}, \ldots\right)\right)=\mathcal{L}\left(\xi_{n 1}+\cdots+\xi_{n z(n)}\right)
$$

\footnotetext{
${ }^{\dagger}$ Supported in part by the Russian Foundation for Basic Research grant 08-01-00078 and by EPSRC grant number EP/D064988/1.

$\ddagger$ Supported in part by EPSRC grant number EP/D064988/1. 
for every $n, z(n) \in \mathbb{N}_{0}$ and $\mathbf{k}_{0}, \mathbf{k}_{1}, \ldots \in \Delta$, where $\xi_{n 1}, \xi_{n 2}, \ldots$ are i.i.d. random variables with distribution $\mathbf{k}_{n}$. Setting

$$
f_{n}(s):=\sum_{j=0}^{\infty} \mathbf{K}_{n}(\{j\}) s^{j}
$$

one can rewrite (1) as

$$
\mathbf{E}\left[s^{Z_{n+1}} \mid Z_{n}=z(n), \overline{\mathbf{K}}=\left(\mathbf{k}_{0}, \mathbf{k}_{1}, \ldots\right)\right]=\left(\sum_{j=0}^{\infty} \mathbf{k}_{n}(\{j\}) s^{j}\right)^{z(n)}, n \geq 0 .
$$

Let $\Omega=\Delta^{\mathbb{N}} \times \mathbb{N}_{0}{ }^{\mathbb{N}}$ be the space of elementary events, endowed with the product $\sigma$-field $\mathcal{F}$ and $\mathbf{P}$ be the corresponding probability measure on the $(\Omega, \mathcal{F})$. The triple $(\Omega, \mathcal{F}, \mathbf{P})$ is be our basic probability space. By $\mathcal{F}_{n}, n \geq 1$, we denote the smallest $\sigma$-field such that projection from $\Omega$ to $\Omega_{n}:=\Delta^{\mathbb{N} \leq \ltimes} \times \mathbb{N}_{0}{ }^{\mathbb{N} \leq n}$ is measurable. $\mathbf{P}_{n}$ is the restriction of $\mathbf{P}$ to $\mathcal{F}_{n}$.

Let

$$
\begin{gathered}
f \stackrel{d}{=} f_{0}, X_{k}:=\ln f_{k-1}^{\prime}(1), \eta_{k}:=f_{k-1}^{\prime \prime}(1)\left(f_{k-1}^{\prime}(1)\right)^{-2}, k \in \mathbb{N}=\{1,2, \ldots\} ; \\
X \stackrel{d}{=} X_{1} ; S_{0}:=0, S_{k}:=X_{1}+\ldots+X_{k}, k \geq 1 .
\end{gathered}
$$

The sequence $\left\{S_{k}, k \geq 0\right\}$ is called the associated random walk of the corresponding branching process in random environment.

Let

$$
T:=\min \left\{k: Z_{k}=0\right\}
$$

and $\tau(m, n):=\min \left\{i \in[m, n]: S_{j} \geq S_{i}, j=m, m+1, \ldots, n\right\}$ be the left-most point of minimum of the random walk $\left\{S_{k}, k \geq 0\right\}$ on the discrete time interval $[m, n]$. In particular we shall write $\tau(n):=$ $\tau(0, n)$ for the left-most point of minimum of the random walk on the discrete time interval $[0, n]$.

Properties of branching processes in random environment are specified to a great extent by the properties of the associated random walk. One of the most important conditions we impose on the characteristics of our branching process in this respect is the following Doney condition:

Assumption $A 1$. There exists a number $0<\rho<1$ such that

$$
\mathbf{P}\left(S_{n}>0\right) \rightarrow \rho \quad \text { as } \quad n \rightarrow \infty .
$$

As it was shown in (7), Condition $A 1$ is equivalent to the classical Spitzer condition

$$
\frac{1}{n} \sum_{k=1}^{n} \mathbf{P}\left(S_{k}>0\right) \rightarrow \rho \quad \text { as } \quad n \rightarrow \infty .
$$

Recall that Assumption $A 1$ implies

$$
n^{-1} \tau(n) \stackrel{d}{\rightarrow} \tau, \quad n \rightarrow \infty
$$

where $\tau$ is a random variable distributed according to the generalized arcsine law with parameter $\rho((11)$, Ch. IV, $\S 20$ ) and the symbol $\stackrel{d}{\rightarrow}$ means convergence in distribution. 
Vatutin and Dyakonova, using the quenched approach, have proved in (16) and (17) conditional limit theorems given $\{T>n\}$ describing the asymptotic behavior, as $n \rightarrow \infty$, of the distribution of the number of particles at moments $Z_{n t}$ and $Z_{\tau(n t)}, t \in(0,1)$, in the branching processes in random environment respecting Assumption $A 1$.

In the present paper we consider the conditioning $\{T=n\}$ and, under Assumption A1 and the quenched approach, study the distribution of the number of particles in our branching process either at moments $n t$, $t \in(0,1)$, or at moments located in a vicinity of points $\tau(n t)$ or $\tau(n t, n)$. (On a notational point, here and in the sequel we understand $n t$ as $[n t]$, the integer part of $n t)$. To formulate our results we need to specify a number of characteristics related with associated random walks.

Let

$$
\gamma_{0}:=0, \quad \gamma_{j+1}:=\min \left(n>\gamma_{j}: S_{n}<S_{\gamma_{j}}\right)
$$

and

$$
\Gamma_{0}:=0, \quad \Gamma_{j+1}:=\min \left(n>\Gamma_{j}: S_{n}>S_{\Gamma_{j}}\right), j \geq 0,
$$

be the strict descending and strict ascending ladder epochs of $\left\{S_{n}, n \geq 0\right\}$. Introduce the functions

$$
\begin{gathered}
V(x)=\left\{\begin{array}{cl}
\sum_{j=0}^{\infty} \mathbf{P}\left(S_{\gamma_{j}} \geq-x\right) & \text { if } x>0, \\
1 & \text { if } x=0, \\
0 & \text { if } x<0,
\end{array}\right. \\
U(x)=\left\{\begin{array}{cl}
1+\sum_{j=1}^{\infty} \mathbf{P}\left(S_{\Gamma_{j}}<x\right) & \text { if } x>0, \\
1 & \text { if } x=0, \\
0 & \text { if } x<0,
\end{array}\right.
\end{gathered}
$$

and set

$$
\Theta(a):=\frac{\sum_{j=a}^{\infty} j^{2} \mathbf{K}(\{j\})}{\left(\sum_{r=0}^{\infty} r \mathbf{K}(\{r\})\right)^{2}}, \quad a \in \mathbb{N} .
$$

Assumption $A 2$. There exist $\varepsilon_{0}>0$ and $a \in \mathbb{N}_{0}$ such that

$$
\begin{aligned}
& \mathbf{E}\left(\log ^{+} \Theta(a)\right)^{\frac{1}{\rho}+\varepsilon_{0}}<\infty \quad \text { and } \quad \mathbf{E}\left[V(X)\left(\log ^{+} \Theta(a)\right)^{1+\varepsilon_{0}}\right]<\infty, \\
& \mathbf{E}\left(\log ^{+} \Theta(a)\right)^{\frac{1}{1-\rho}+\varepsilon_{0}}<\infty \quad \text { and } \quad \mathbf{E}\left[U(-X)\left(\log ^{+} \Theta(a)\right)^{1+\varepsilon_{0}}\right]<\infty .
\end{aligned}
$$

One can find in (1) and (16) more details demonstrating the importance of these conditions.

Finally, we impose a (rather specific) condition on the form of the probability generating functions of the underlying branching process in random environment.

Assumption $A 3$. The random offspring generating functions $f_{n}(s), n=0,1, \ldots$, are fractional-linear, i.e., they have with probability 1 the following form

$$
f_{n}(s)=r_{n}+\left(1-r_{n}\right) \frac{q_{n}}{1-p_{n} s}
$$

where $p_{n}+q_{n}=1, p_{n} q_{n}>0$.

It turns out that, many (but not all) of the forthcoming results in this paper can be proved for the branching processes in random environment respecting Assumptions $A 1-A 2$ only. However, the main 
advantage we gain by imposing Assumption $A 3$ is that if we let

$$
\begin{aligned}
f_{k, n}(s) & :=f_{k}\left(f_{k+1}\left(\ldots\left(f_{n-1}(s)\right) \ldots\right)\right), 0 \leq k \leq n-1, f_{n, n}(s):=s \\
f_{n, m}(s) & :=f_{n-1}\left(f_{n-2}\left(\ldots\left(f_{m}(s)\right) \ldots\right)\right), n \geq m+1
\end{aligned}
$$

and denote

$$
\eta_{j+1}:=\frac{f_{j}^{\prime \prime}(1)}{\left(f_{j}^{\prime}(1)\right)^{2}}, b_{m}:=\frac{1}{2} \sum_{j=0}^{m-1} \eta_{j+1} e^{-S_{j}}
$$

(noting that both are positive quantities), then Assumption $A 3$ implies (see, for instance, (10)) that for $m=0,1, \ldots$ and $s \in[0,1]$,

$$
\frac{1}{1-f_{0, m}(s)}=\frac{e^{-S_{m}}}{1-s}+b_{m}
$$

Later on we need various probability measures and (random) conditional expectations specified on the measurable space $(\Omega, \mathcal{F})$. To distinguish them we use the symbols $\mathbf{E}$ and $\mathbf{P}$ to denote the expectation and probability law generated by the initial measure on the tuples

$$
\left(f_{0}, f_{1}, \ldots, f_{n}, \ldots ; Z_{0}, Z_{1}, \ldots, Z_{n}, \ldots\right)
$$

(there may be some occasional mild abuse of this notation however we do not anticipate that it will lead to confusion). Besides we write for brevity $\mathbf{E}_{\overline{\mathbf{K}}}[\cdot]$ and $\mathbf{P}_{\overline{\mathbf{K}}}[\cdot]$ for the conditional expectation $\mathbf{E}[\cdot \mid \overline{\mathbf{K}}]$ and conditional probability $\mathbf{P}[\cdot \mid \overline{\mathbf{K}}]$ with respect to the $\sigma$-field generated by the infinite-dimensional vector $\overline{\mathbf{K}}=\left(\mathbf{K}_{0}, \mathbf{K}_{1}, \ldots\right)$. Along with the basic probability space $(\Omega, \mathcal{F}, \mathbf{P})$ we deal with two (independent) copies $\left(\Omega^{-}, \mathcal{F}^{-}, \mathbf{P}^{-}\right)$and $\left(\Omega^{+}, \mathcal{F}^{+}, \mathbf{P}^{+}\right)$. Denote by $\left\{f_{n}^{-}, n \geq 0\right\}$ and $\left\{f_{n}^{+}, n \geq 0\right\}$ two sequences of the random environment and by $\left\{S_{n}^{-}, n \geq 0\right\}$ and $\left\{S_{n}^{+}, n \geq 0\right\}$ the corresponding associate random walks specified on $\left(\Omega^{-}, \mathcal{F}^{-}, \mathbf{P}^{-}\right)$and $\left(\Omega^{+}, \mathcal{F}^{+}, \mathbf{P}^{+}\right)$, respectively. Later on any characteristics or random variables related with $\left\{f_{n}^{-}, n \geq 0\right\}$ and $\left\{f_{n}^{+}, n \geq 0\right\}$ are superscripted with the symbols - or + , respectively. Following this practice, we write

$$
\Gamma^{-}=\min \left\{n \geq 1: S_{n}^{-} \geq 0\right\}
$$

and

$$
\gamma^{+}=\min \left\{n \geq 1: S_{n}^{+}<0\right\} .
$$

We also study various properties of the pair of branching processes in random environment given the event

$$
\mathcal{A}_{k, p}:=\left\{\Gamma^{-}>k, \gamma^{+}>p\right\} .
$$

Set $D=\sum_{j=1}^{\infty} \mathbf{P}\left(S_{j}=0\right)$. In addition to the measures $\mathbf{P}^{-}$and $\mathbf{P}^{+}$we define measures $\hat{\mathbf{P}}^{-}$and $\hat{\mathbf{P}}^{+}$ on $\left(\Omega^{-}, \mathcal{F}^{-}\right)$and $\left(\Omega^{+}, \mathcal{F}^{+}\right)$whose restrictions $\hat{\mathbf{P}}_{k}^{-}$and $\hat{\mathbf{P}}_{k}^{+}$on the $\sigma$-algebras $\mathcal{F}_{k}^{-}$and $\mathcal{F}_{k}^{+}, k \in \mathbb{N}$ are specified by

$$
\begin{aligned}
\hat{\mathbf{P}}_{k}^{-}\left(\mathcal{A}^{-}\right) & =e^{D} \int_{\mathcal{A}^{-}} U\left(-S_{k}^{-}\right) I\left\{\Gamma^{-}>k\right\} d \mathbf{P}^{-}, \mathcal{A}^{-} \in \mathcal{F}_{k}^{-}, \\
\hat{\mathbf{P}}_{k}^{+}\left(\mathcal{A}^{+}\right) & =\int_{\mathcal{A}^{+}} V\left(S_{k}^{+}\right) I\left\{\gamma^{+}>k\right\} d \mathbf{P}^{+}, \mathcal{A}^{+} \in \mathcal{F}_{k}^{+} .
\end{aligned}
$$


One can check (see, (17)) that the sequences $\left\{\hat{\mathbf{P}}_{k}^{-}, k \in \mathbb{N}\right\},\left\{\hat{\mathbf{P}}_{k}^{-}, k \in \mathbb{N}\right\}$ consist of well-defined and consistent probability measures. The probabilistic sense of these measures is rather transparent: the restriction of $\hat{\mathbf{P}}^{-}$to $\mathcal{F}_{k}^{-}$is concentrated only on the realizations of the environment whose associated random walks are negative for the first $k$ steps (except the starting point) while the restriction of $\hat{\mathbf{P}}^{+}$ to $\mathcal{F}_{k}^{+}$is concentrated only on the realizations of the environment whose associated random walks are nonnegative for the first $k$ steps. Indeed, in an appropriate sense, $\hat{\mathbf{P}}^{+}$and $\hat{\mathbf{P}}^{-}$may be thought of as the random walks $S^{+}$and $S^{-}$conditioned to stay positive. See for example (5).

Further, on the measurable space $\left(\Omega^{-} \times \Omega^{+}, \mathcal{F}^{-} \times \mathcal{F}^{+}\right)$we specify the probability measure $\hat{\mathbf{P}}:=$ $\hat{\mathbf{P}}^{-} \times \hat{\mathbf{P}}^{+}$, whose projection on the elements of the $\sigma$-algebra $\mathcal{F}_{k}^{-} \times \mathcal{F}_{p}^{+}$is given by

$$
\hat{\mathbf{P}}(\mathcal{A})=e^{D} \int_{\mathcal{A}} U\left(-S_{k}^{-}\right) V\left(S_{p}^{+}\right) I\left\{\mathcal{A}_{k, p}\right\} d\left(\mathbf{P}^{-} \times \mathbf{P}^{+}\right), \mathcal{A} \in \mathcal{F}_{k}^{-} \times \mathcal{F}_{p}^{+}
$$

(see (17) for more detailed description of this measure).

With the notation above in hand we list for further references some results established in (16) before moving to our main results.

1) Under Assumptions $A 1-A 2$ for any $R \in \mathbb{N}_{0}$ there exists the limit

$$
q_{R}^{+}:=\lim _{n \rightarrow \infty} f_{R, n}^{+}(0)<1 \hat{\mathbf{P}}^{+} \text {- a.s. }
$$

(later on we write for brevity $q^{+}$for $q_{0}^{+}$);

2) the tuple of random functions

$$
\zeta_{l, m}^{-}(s):=\frac{1-f_{l, m}^{-}(s)}{e^{S_{l}^{-}-S_{m}^{-}}}, m \in \mathbb{N}_{0}, l \geq m+1,
$$

is such that $\hat{\mathbf{P}}$ - a.s. the limit

$$
\zeta_{\infty, m}^{-}(s):=\lim _{l \rightarrow \infty} \zeta_{l, m}^{-}(s)
$$

exists and is positive and less than 1 for any $s \in[0,1)$.

For brevity we set $\zeta_{l}^{-}(s):=\zeta_{l, 0}^{-}(s)$ and $\zeta^{-}(s):=\zeta_{\infty, 0}^{-}(s)$. Observe that

$$
\zeta:=\lim _{\min (l, n-l) \rightarrow \infty} \zeta_{l}^{-}\left(f_{0, n-l}^{+}(0)\right)=\zeta^{-}\left(q^{+}\right)
$$

exists $\hat{\mathbf{P}}-$ a.s. and, moreover, $\zeta \in(0,1]$ with probability 1 .

\section{Main results}

Now we are ready to formulate the main results of the present paper. Below our two main theorems we offer some intuition as to their interpretation. 
Theorem 1 Suppose that $A 1-A 3$ hold. Then for any $R \in \mathbb{Z}$, any $t \in(0,1)$, and $s \in(0,1]$

1)

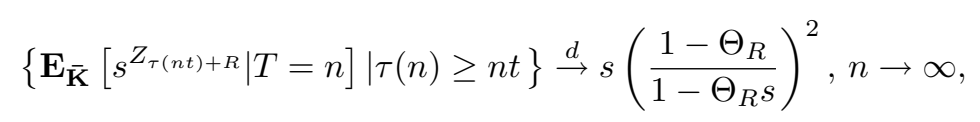

where

$$
\Theta_{R}=\left\{\begin{array}{cc}
\zeta^{-}\left(f_{0, R}^{+}(0)\right) e^{-S_{R}^{+}} & \text {if } \quad R \geq 0, \\
\zeta_{\infty, R}^{-}(0) e^{-S_{R}^{-}} & \text {if } \quad R<0,
\end{array}\right.
$$

and $\Theta_{R} \in(0,1)$ with probability 1;

2)

$$
\left\{\mathbf{E}_{\overline{\mathbf{K}}}\left[s^{Z_{\tau(n t, n)+R}} \mid T=n\right] \mid \tau(n)<n t\right\} \stackrel{d}{\rightarrow} s\left(\frac{1-\theta_{R}}{1-\theta_{R} s}\right)^{2}, n \rightarrow \infty,
$$

where

$$
\theta_{R}=\left\{\begin{array}{ccc}
q_{R}^{+} & \text {if } & R \geq 0 \\
f_{R, 0}^{-}\left(q^{+}\right) & \text {if } & R<0
\end{array}\right.
$$

and $\theta_{R} \in(0,1)$ with probability 1 .

The next theorem deals with the distribution of the number of particles at moments $n t, 0<t<1$. Let

$$
O_{m, n}:=\frac{1-f_{0, n}(0)}{1-f_{m, n}(0)} f_{m, n}(0) b_{m}
$$

Theorem 2 Suppose that Assumptions $A 1-A 3$ hold. Then, for any $t \in(0,1)$ and $\lambda \in(0, \infty)$

$$
\mathbf{E}_{\overline{\mathbf{K}}}\left[\exp \left\{-\lambda \frac{Z_{n t}}{O_{n t, n}}\right\} \mid T=n\right] \stackrel{p}{\rightarrow} \frac{1}{(1+\lambda)^{2}} \text { as } n \rightarrow \infty .
$$

It is necessary to note that despite of the unique form of the limit in the cases $\{\tau(n) \geq n t\}$ and $\{\tau(n)<n t\}$, the behavior of the scaling function $O_{n t, n}$ as $n \rightarrow \infty$ is different in the two theorems. Later on in Lemmas 20 and 21 (see also Remark 23) we shall see that on the set $\tau(n) \geq n t$ the random variable $O_{n t, n} e^{S_{\tau(n t)}-S_{n t}}$ converges in distribution, as $n \rightarrow \infty$, to a random variable being finite and positive with probability 1 . Thus, the normalization in Theorem 1 is, essentially, specified by the past behavior of the associated random walk. On the other hand, given $\tau(n)<n t$ the random variable $O_{n t, n} e^{S_{\tau(n t, n)}-S_{n t}}$ converges in distribution, as $n \rightarrow \infty$, to a random variable being finite and positive with probability 1 . Thus, the scaling in in Theorem 2 is, essentially, specified by the future behavior of the associated random walk.

This fact allows us to give the following non-rigorous interpretation of our results. If the process dies out at a distant moment $T=n$ then it happens not as a unique catastrophic event. Before the extinction moment the evolution of the process consists of a number of "bad" periods where the size of the population is small. According to Theorem 11, such periods are located in the vicinities of random points $\tau(n t) I\{\tau(n) \geq n t\}$ and $\tau(n t, n) I\{\tau(n)<n t\}$. On the other hand, at nonrandom points $n t, t \in(0,1)$, the size of the population is, by Theorem 2 big. Hence $\log Z_{n t}$ grows like $S_{n t}-S_{\tau(n t)}$ if $\tau(n)>n t$ and 
like $S_{n t}-S_{\tau(n t, n)}$ if $\tau(n)<n t$. Thus, the process $\left\{Z_{t T}, 0 \leq t \leq 1\right\}$ dies by passing through a number of bottlenecks and favorable periods.

The remainder of the paper consists of two sections. The first deals with the proof of Theorem 1 and the second with the proof of Theorem 2 .

\section{Proof of Theorem 1}

The proof of Theorem 1 is given right at the very end of this section. We must first pass through a large number of technical results.

We begin by setting

$$
\Delta_{m, n}:=\left(\frac{1-f_{m, n-1}(0)}{1-f_{m, n}(0)}\right)^{2}\left(\frac{1-f_{0, n}(0)}{1-f_{0, n-1}(0)}\right)^{2}=\frac{O_{m, n}^{2}}{O_{m, n-1}^{2}} \frac{f_{m, m-1}^{2}(0)}{f_{m, n}^{2}(0)}
$$

and justifying the following key estimate.

Lemma 3 Under Assumption A3 for any $1 \leq m<n$

$$
\frac{1}{1+(1-s) O_{m, n-1}} \Delta_{m, n}^{-1} \leq \mathbf{E}_{\overline{\mathbf{K}}}\left[s^{Z_{m}} \mid T=n\right] \leq \frac{1}{1+(1-s) O_{m, n}} \Delta_{m, n} .
$$

Proof. Clearly,

$$
\begin{aligned}
\mathbf{E}_{\overline{\mathbf{K}}}\left[s^{Z_{m}} \mid T=n\right] & =\frac{\mathbf{E}_{\overline{\mathbf{K}}}\left[s^{Z_{m}} ; T=n\right]}{\mathbf{P}_{\overline{\mathbf{K}}}(T=n)} \\
& =\frac{\mathbf{E}_{\overline{\mathbf{K}}}\left[s^{Z_{m}} ; Z_{n}=0\right]-\mathbf{E}_{\overline{\mathbf{K}}}\left[s^{Z_{m}} ; Z_{n-1}=0\right]}{f_{0, n}(0)-f_{0, n-1}(0)} \\
& =\frac{f_{0, m}\left(s f_{m, n}(0)\right)-f_{0, m}\left(s f_{m, n-1}(0)\right)}{f_{0, m}\left(f_{m, n}(0)\right)-f_{0, m}\left(f_{m, n-1}(0)\right)} .
\end{aligned}
$$

Hence, using the Mean Value Theorem and the monotonicity properties of $f_{0, m}^{\prime}(s)$ in $s$ and $f_{m, N}(0)$ in $N$ we get

$$
s \frac{f_{0, m}^{\prime}\left(s f_{m, n-1}(0)\right)}{f_{0, m}^{\prime}\left(f_{m, n}(0)\right)} \leq \mathbf{E}_{\overline{\mathbf{K}}}\left[s^{Z_{m}} \mid T=n\right] \leq s \frac{f_{0, m}^{\prime}\left(s f_{m, n}(0)\right)}{f_{0, m}^{\prime}\left(f_{m, n-1}(0)\right)} .
$$

It is easy to conclude from (6) that under Assumption $A 3$

$$
f_{0, m}^{\prime}(s)=\frac{e^{-S_{m}}}{(1-s)^{2}}\left(1-f_{0, m}(s)\right)^{2} .
$$

Therefore,

$$
\frac{f_{0, m}^{\prime}\left(s f_{m, n}(0)\right)}{f_{0, m}^{\prime}\left(f_{m, n-1}(0)\right)}=\frac{\left(1-f_{0, m}\left(s f_{m, n}(0)\right)\right)^{2}}{\left(1-s f_{m, n}(0)\right)^{2}} \frac{\left(1-f_{m, n-1}(0)\right)^{2}}{\left(1-f_{0, n-1}(0)\right)^{2}}
$$


Further, again making use of $(6)$, we have for $s \in[0,1]$

$$
\begin{aligned}
\frac{1-f_{0, m}\left(s f_{m, n}(0)\right)}{1-s f_{m, n}(0)} & =\frac{1}{e^{-S_{m}}+\left(1-s f_{m, n}(0)\right) b_{m}} \\
& =\frac{1}{e^{-S_{m}}+\left(1-f_{m, n}(0)\right) b_{m}+(1-s) f_{m, n}(0) b_{m}} \\
& =\frac{1}{\left(1-f_{m, n}(0)\right)\left(1-f_{0, n}(0)\right)^{-1}+(1-s) f_{m, n}(0) b_{m}} \\
& =\frac{1-f_{0, n}(0)}{1-f_{m, n}(0)} \times \frac{1}{1+(1-s) O_{m, n}}
\end{aligned}
$$

where the third equality follows from the first equality when $s=1$. As a result we have

$$
\frac{f_{0, m}^{\prime}\left(s f_{m, n}(0)\right)}{f_{0, m}^{\prime}\left(f_{m, n-1}(0)\right)}=\frac{1}{1+(1-s) O_{m, n}} \Delta_{m, n}
$$

Similarly,

$$
\frac{f_{0, m}^{\prime}\left(s f_{m, n-1}(0)\right)}{f_{0, m}^{\prime}\left(f_{m, n}(0)\right)}=\frac{1}{1+(1-s) O_{m, n-1}} \Delta_{m, n}^{-1}
$$

The lemma is proved.

To proceed further we need to formulate for future reference several known statements. In the next two lemmas recall that $\mathcal{A}_{n, r}$ was defined in (7).

Lemma 4 ((16), Lemma 3) Let Assumption $A 1$ be valid and let $T_{l, p}, l, p \in \mathbb{N}$, be a tuple of uniformly bounded random variables such that, for any pair $l, p$ the random variable $T_{l, p}$ is measurable with respect to the $\sigma$-algebra $\mathcal{F}_{l}^{-} \times \mathcal{F}_{p}^{+}$. Then

$$
\lim _{\min (n, r) \rightarrow \infty} \mathbf{E}\left[T_{l, p} \mid \mathcal{A}_{n, r}\right]=\hat{\mathbf{E}}\left[T_{l, p}\right] .
$$

More generally, if the tuple $\left\{T_{n, r}, n, r \in \mathbb{N}\right\}$ consists of the uniformly bounded random variables which are adopted to the flow of the $\sigma$-algebras $\left\{\mathcal{F}_{n}^{-} \times \mathcal{F}_{r}^{+}\right\}_{n \geq 1, r \geq 1}$, and $\lim _{\min (n, r) \rightarrow \infty} T_{n, r}=:$ T exists $\hat{\mathbf{P}}-$ a.s. then

$$
\lim _{\min (n, r) \rightarrow \infty} \mathbf{E}\left[T_{n, r} \mid \mathcal{A}_{n, r}\right]=\hat{\mathbf{E}}[T] .
$$

Lemma 5 ((16), Lemma 4) Let Assumption $A 1$ be valid and let $T$ and $T_{l, p}, l, p \in \mathbb{N}$ be a tuple of random variables meeting the conditions of Lemma 4 . If $T_{l, p}^{*}, l, p \in \mathbb{N}$ is a tuple of uniformly bounded random variables such that for any pair $l, p$ the random variable, $T_{l, p}^{*}$ is measurable with respect to the $\sigma$-algebra $\mathcal{F}_{l}^{-} \times \mathcal{F}_{p}^{+}$and

$$
\mathbf{E}\left[T_{\tau(n), n}^{*} \mid \tau(n)=l\right]=\mathbf{E}\left[T_{l, n-l} \mid \mathcal{A}_{l, n-l}\right],
$$

then

$$
\lim _{n \rightarrow \infty} \mathbf{E}\left[T_{\tau(n), n}^{*}\right]=\hat{\mathbf{E}}[T] .
$$


For $0 \leq m \leq n$ set

$$
\alpha_{n}(m):=\frac{1-f_{0, m}(0)}{1-f_{0, n}(0)}, \quad \beta_{n}(m):=\frac{1-f_{0, n}(0)}{e^{S_{m}}\left(1-f_{m, n}(0)\right)} .
$$

Clearly, $\alpha_{n}(m) \geq 1, \beta_{n}(m) \leq 1$, and, in addition,

$$
\begin{aligned}
O_{m, n} & =\frac{1}{\alpha_{n}(m)} \times \frac{f_{m, n}(0)}{1-f_{m, n}(0)}-f_{m, n}(0) \beta_{n}(m) \\
& =f_{m, n}(0) \beta_{n}(m)\left(\frac{e^{S_{m}}}{1-f_{0, m}(0)}-1\right) .
\end{aligned}
$$

Lemma 6 (([16), Lemma 16) Assume that $A 1-A 2$ hold. Then for $R \in \mathbb{Z}$ and any $\varepsilon>0$

$$
\limsup _{R, n \rightarrow \infty} \mathbf{P}\left(\alpha_{n}(\tau(n)+R)>1+\varepsilon\right)=0 .
$$

Remark 7 Since the random variable $\alpha_{n}(\tau(n)+R)$ is not defined for $\tau(n)+R<0$ or $\tau(n)+R>n$ we should formally write the statement of the lemma as

$$
\limsup _{R, n \rightarrow \infty} \mathbf{P}\left(\alpha_{n}(\tau(n)+R)>1+\varepsilon ; n \geq \tau(n)+R \geq 0\right)=0
$$

However, by the generalized arcsine law for each fixed $R \in Z$

$$
\lim _{n \rightarrow \infty} \mathbf{P}(\tau(n)+R \notin[0, n])=0 .
$$

For this reason here and in what follows we agree to treat $\alpha_{n}(\tau(n)+R)$ as $\alpha_{n}(0)$ if $\tau(n)+R<0$ and $\alpha_{n}(n)$ if $\tau(n)+R>n$. Similar agreement will be kept for other functions which involve $\tau(n)+R$, $\tau(n t)+R$ or $\tau(n t, n)+R$.

Lemma 8 ((16), Corollary 3) Assume that A1-A2 hold. For any $t \in(0,1]$ and $\varepsilon>0$

$$
\limsup _{n \rightarrow \infty} \mathbf{P}\left(\alpha_{n}(n t)>1+\varepsilon \mid \tau(n)<n t\right)=0 .
$$

The next statement complements Lemmas 6 and 8

Lemma 9 Assume that $A 1-A 2$ hold. Then for $R \in \mathbb{Z}$, any $t \in(0,1]$, and any $\varepsilon>0$

$$
\limsup _{R, n \rightarrow \infty} \mathbf{P}\left(\alpha_{n}(\tau(n t, n)+R)>1+\varepsilon \mid \tau(n)<n t\right)=0 .
$$

Proof. First we note that $\alpha_{n}(m) \downarrow 1$ as $m \uparrow n$. Hence

$$
\begin{aligned}
\alpha_{n}(\tau(n t, n)+R) I\{n t \leq \tau(n t, n)+R \leq n\} & \leq \alpha_{n}(n t) I\{n t \leq \tau(n t, n)+R \leq n\} \\
& \leq \alpha_{n}(n t) .
\end{aligned}
$$


Therefore,

$$
\begin{aligned}
\mathbf{P}\left(\alpha_{n}(\tau(n t, n)+R)>1+\varepsilon ; \tau(n)<n t\right) \leq & \mathbf{P}\left(\alpha_{n}(n t)>1+\varepsilon ; \tau(n)<n t\right) \\
& +\mathbf{P}(\tau(n t, n)+R \notin[n t, n]) .
\end{aligned}
$$

Observing that $\{\tau(n t, n)+R>n\}=\oslash$ for $R<0$ and $\{\tau(n t, n)+R<n t\}=\oslash$ for $R>0$ and recalling the generalized arcsine law (which, under Assumption $A 1$, holds for $n^{-1} \tau(n t, n)$ (see (11))) we see that for each fixed $R$

$$
\lim _{n \rightarrow \infty} \mathbf{P}(\tau(n t, n)+R \notin[n t, n])=0 .
$$

Passing now to the limit as $n \rightarrow \infty$ in the both sides of 22 and recalling Lemma 8 we see that

$$
\lim \sup _{n \rightarrow \infty} \mathbf{P}\left(\alpha_{n}(\tau(n t, n)+R)>1+\varepsilon ; \tau(n)<n t\right)=0 .
$$

Hence the statement of the lemma follows.

Lemma 10 Assume that $A 1-A 2$ hold. Then for any $R \in \mathbb{Z}$, any $t \in(0,1)$, and $N \in\{n-1, n\}$

$$
\left\{f_{\tau(n t, n)+R, N}(0) \mid \tau(n)<n t\right\} \stackrel{d}{\rightarrow} \theta_{R} \text { as } n \rightarrow \infty
$$

where $\theta_{R}$ is the same as in Theorem 1 .

Proof. Consider first $R<0$. Clearly,

$$
\begin{aligned}
& \mathbf{E}\left[e^{-\lambda f_{\tau(n t, n)+R, N}(0)} ; \tau(n)<n t\right] \\
= & \mathbf{E}\left[e^{-\lambda f_{\tau(n t, n)+R, N}(0)}\right]-\mathbf{E}\left[e^{-\lambda f_{\tau(n t, n)+R, N}(0)} ; \tau(n) \geq n t\right] .
\end{aligned}
$$

Introduce two independent environmental sequences $\left\{f_{n}^{-}, n \geq 0\right\}$ and $\left\{f_{n}^{+}, n \geq 0\right\}$ and the respective associated random walks $\left\{S_{n}^{-}, n \geq 0\right\}$ and $\left\{S_{n}^{+}, n \geq 0\right\}$. In the notation of Lemmas 4 and 5 we set

$$
T_{\tau(n(1-t)), n(1-t)}^{*}:=e^{-\lambda f_{\tau(n(1-t))+R, N-n(1-t)}(0)}, T_{l, n-l}:=e^{-\lambda f_{R, 0}^{-}\left(f_{0, N-n(1-t)-l}^{+}(0)\right)} .
$$

Observing that

$$
e^{-\lambda f_{\tau(n(1-t))+R, N-n(1-t)}(0)} \stackrel{d}{=} e^{-\lambda f_{\tau(n t, n)+R, N}(0)}
$$

and

$$
f_{R, 0}^{-}\left(f_{0, N-n(1-t)-l}(0)\right) \rightarrow f_{R, 0}^{-}\left(q^{+}\right)
$$

$\hat{\mathbf{P}}-$ a.s. as $\min (l, N-n(1-t)-l) \rightarrow \infty$ we conclude by Lemma 5 and the generalized arcsine law that

$$
\begin{aligned}
\mathbf{E}\left[e^{-\lambda f_{\tau(n t, n)+R, N}(0)}\right] & =\mathbf{E}\left[e^{-\lambda f_{\tau(n(1-t))+R, N-n(1-t)}(0)}\right] \\
& \rightarrow \hat{\mathbf{E}}\left[e^{-\lambda f_{R, 0}^{-}\left(q^{+}\right)}\right], n \rightarrow \infty
\end{aligned}
$$


Further we have

$$
\begin{aligned}
\mathbf{E} & {\left[e^{-\lambda f_{\tau(n t, n)+R, N}(0)} ; \tau(n) \geq n t\right]=\mathbf{E}\left[e^{-\lambda f_{\tau(n t, n)+R, N}(0)} ; \tau(n) \geq n t\right] } \\
& =\sum_{j=n t}^{n} \mathbf{E}\left[e^{-\lambda f_{\tau(n)+R, N}(0)} ; \tau(n)=j\right] \\
& =\sum_{j=n t}^{n} \mathbf{E}\left[e^{-\lambda f_{j+R, N}(0)} ; \min _{0 \leq r<j} S_{r}>S_{j}, \min _{j+1 \leq r \leq n} S_{r} \geq S_{j}\right] \\
& =\sum_{j=n t}^{n} \mathbf{E}\left[e^{-\lambda f_{R, 0}^{-}\left(f_{0, N-j}^{+}(0)\right)} ; A_{j, n-j}\right] \\
& =\sum_{j=n t}^{n} \mathbf{E}\left[e^{-\lambda f_{R, 0}^{-}\left(f_{0, N-j}^{+}(0)\right)} \mid A_{j, n-j}\right] \mathbf{P}(\tau(n)=j)
\end{aligned}
$$

and since $f_{R, 0}^{-}\left(f_{0, N-j}^{+}(0)\right) \rightarrow f_{R, 0}^{-}\left(q^{+}\right) \hat{\mathbf{P}}-$ a.s as $n-j \rightarrow \infty$ we have by Lemma 4 that

$$
\lim _{n-j \rightarrow \infty} \mathbf{E}\left[e^{-\lambda f_{R, N-j}(0)} \mid A_{j, n-j}\right]=\hat{\mathbf{E}}\left[e^{-\lambda f_{R, 0}^{-}\left(q^{+}\right)}\right] .
$$

This and the generalized arcsine law give

$$
\lim _{n \rightarrow \infty} \mathbf{E}\left[e^{-\lambda f_{\tau(n t, n)+R, N}(0)} ; \tau(n) \geq n t\right]=\hat{\mathbf{E}}\left[e^{-\lambda f_{R, 0}^{-}\left(q^{+}\right)}\right] \mathbf{P}(\tau \geq t)
$$

Thus,

$$
\lim _{n \rightarrow \infty} \mathbf{E}\left[e^{-\lambda f_{\tau(n t, n)+R, N}(0) I\{\tau(n t, n)+R \geq 0\}} ; \tau(n)<n t\right]=\hat{\mathbf{E}}\left[e^{-\lambda f_{R, 0}^{-}\left(q^{+}\right)}\right] \mathbf{P}(\tau \leq t)
$$

proving the lemma for $R<0$.

The case $R \geq 0$ can be treated in a similar way by observing that, in this case, for all $j \leq N-R$

$$
\mathbf{E}\left[e^{-\lambda f_{\tau(n)+R, N}(0)} ; \tau(n)=j\right]=\mathbf{E}\left[e^{\left.-\lambda f_{R, N-j}^{+}(0)\right)} \mid A_{j, n-j}\right] \mathbf{P}(\tau(n)=j)
$$

and that (11) holds.

In what follows we need some properties of the random variable $\beta_{n}(m)$.

Lemma 11 ((16), Lemma 17) Assume that $A 1-A 2$ hold. For any $\varepsilon>0$ and $N \in\{n-1, n\}$

$$
\limsup _{R, n \rightarrow \infty} \mathbf{P}\left(\beta_{N}(\tau(n)+R)>\varepsilon\right)=0 .
$$

Lemma 12 ((16), Corollary 4) Assume that $A 1-A 2$ hold. For any $t \in(0,1)$ and $\varepsilon>0$

$$
\begin{aligned}
& \limsup _{n \rightarrow \infty} \mathbf{P}\left(\beta_{n}(n t)<1-\varepsilon ; \tau(n) \geq n t\right)=0, \\
& \limsup _{n \rightarrow \infty} \mathbf{P}\left(\beta_{n}(n t)>\varepsilon ; \tau(n)<n t\right)=0 .
\end{aligned}
$$


Lemma 13 Assume that $A 1-A 2$ hold. For any $R \in \mathbb{Z}$, any $t \in(0,1)$, and $N \in\{n-1, n\}$

$$
\left\{O_{\tau(n t, n)+R, N} \mid \tau(n)<n t\right\} \stackrel{d}{\rightarrow} \frac{\theta_{R}}{1-\theta_{R}} .
$$

Proof. We have

$$
O_{\tau(n t, n)+R, N}=\frac{1}{\alpha_{N}(\tau(n t, n)+R)} \times \frac{f_{\tau(n t, n)+R, N}(0)}{1-f_{\tau(n t, n)+R, N}(0)}-f_{\tau(n t, n)+R, N}(0) \beta_{N}(\tau(n t, n)+R) .
$$

By Lemmas 9 and 10

$$
\frac{1}{\alpha_{N}(\tau(n t, n)+R)} \times \frac{f_{\tau(n t, n)+R, N}(0)}{1-f_{\tau(n t, n)+R, N}(0)} \stackrel{d}{\rightarrow} \frac{\theta_{R}}{1-\theta_{R}}, n \rightarrow \infty .
$$

Further, by 26]

$$
\begin{aligned}
& \beta_{N}(\tau(n t, n)+R) I\{\tau(n)<n t \leq \tau(n t, n)+R\} \\
& \quad \leq \beta_{N}(n t) I\{\tau(n)<n t \leq \tau(n t, n)+R\} \\
& \quad \leq \beta_{N}(n t) I\{\tau(n)<n t\} \stackrel{p}{\rightarrow} 0
\end{aligned}
$$

as $n \rightarrow \infty$. Using (28) and (30) to evaluate (27) proves the lemma.

Lemma 14 Assume that $A 1-A 2$ hold. For any $t \in(0,1)$, any fixed $R \in \mathbb{Z}$, and $\varepsilon>0$

$$
\limsup _{n \rightarrow \infty} \mathbf{P}\left(\beta_{n}(\tau(n t)+R)<1-\varepsilon ; \tau(n) \geq n t\right)=0 .
$$

Proof. Since

$$
e^{S_{m}}\left(1-f_{m, n}(s)\right) \leq e^{S_{m+1}}\left(1-f_{m+1, n}(s)\right)
$$

for any $m<n$, the elements of the sequence $\left\{\beta_{n}(m), 0 \leq m \leq n\right\}$ are monotone decreasing in $m$ for any fixed $n$. On the other hand, for any $\delta>0$ there exists $\varepsilon_{1}>0$ such that

$$
\mathbf{P}\left(\tau(n t)+R>n t\left(1-\varepsilon_{1}\right)\right)<\delta
$$

for all $n \geq n_{0}=n_{0}\left(\delta, \varepsilon_{1}\right)$. Thus, we have for all $n \geq n_{0}$

$$
\begin{aligned}
& \mathbf{P}\left(\beta_{n}(\tau(n t)+R)<1-\varepsilon ; \tau(n) \geq n t\right) \\
& \quad \leq \delta+\mathbf{P}\left(\beta_{n}\left(n t\left(1-\varepsilon_{1}\right)\right)<1-\varepsilon ; \tau(n) \geq n t\right) \\
& \quad \leq \delta+\mathbf{P}\left(\beta_{n}\left(n t\left(1-\varepsilon_{1}\right)\right)>1-\varepsilon ; \tau(n) \geq n t\left(1-\varepsilon_{1}\right)\right)
\end{aligned}
$$

To complete the proof it remains to recall 25].

Lemma 15 Assume that $A 1-A 2$ hold. For any $R \in \mathbb{Z}$, any $t \in(0,1)$, and $N \in\{n-1, n\}$

$$
\left\{f_{\tau(n t)+R, N}(0) \mid \tau(n) \geq n t\right\} \stackrel{d}{\rightarrow} 1, n \rightarrow \infty .
$$


Proof. For a fixed $\varepsilon \in(0,1)$ introduce the event

$$
\mathcal{H}_{n}(\varepsilon)=\{\omega:\{\tau(n t), \tau(n t)+R, \tau(n)\} \cap[n t(1-\varepsilon), n t(1+\varepsilon)]=\oslash\}
$$

and let $\overline{\mathcal{H}}_{n}(\varepsilon)$ be the complement of $\mathcal{H}_{n}(\varepsilon)$. By the generalized arcsine law

$$
\begin{aligned}
\lim _{\varepsilon \downarrow 0} \lim _{n \rightarrow \infty} \mathbf{P}\left(\overline{\mathcal{H}}_{n}(\varepsilon)\right) \leq & \lim _{\varepsilon \downarrow 0} \lim _{n \rightarrow \infty} \mathbf{P}(\tau(n t) \in[n t(1-\varepsilon), n t(1+\varepsilon)]) \\
& +\lim _{\varepsilon \downarrow 0} \lim _{n \rightarrow \infty} \mathbf{P}(\tau(n t)+R \in[n t(1-\varepsilon), n t(1+\varepsilon)]) \\
& +\lim _{\varepsilon \downarrow 0} \lim _{n \rightarrow \infty} \mathbf{P}(\tau(n) \in[n t(1-\varepsilon), n t(1+\varepsilon)]) \\
= & 0 .
\end{aligned}
$$

Hence, to prove the lemma it is suffices to show that

$$
\left\{f_{\tau(n t)+R, N}(0) \mid \mathcal{H}_{n}(\varepsilon) ; \tau(n) \geq n t\right\} \stackrel{d}{\rightarrow} 1, n \rightarrow \infty .
$$

Clearly,

$$
\begin{aligned}
& \left(1-f_{\tau(n t)+R, N}(0)\right) I\left\{\mathcal{H}_{n}(\varepsilon) ; \tau(n) \geq n t\right\} \\
& \leq\left(1-f_{\tau(n t)+R, \tau(n)}(0)\right) I\left\{\mathcal{H}_{n}(\varepsilon) ; \tau(n)>n t(1+\varepsilon)\right\} \\
& \leq e^{S_{\tau(n)}-S_{\tau(n t)+R}} I\left\{\mathcal{H}_{n}(\varepsilon) ; \tau(n)>n t(1+\varepsilon)\right\} \leq e^{S_{\tau(n)}-S_{\tau(n t)}} I\left\{\mathcal{H}_{n}(\varepsilon) ; \tau(n)>n t(1+\varepsilon)\right\} \\
& \leq e^{S_{\tau(n)}-S_{\tau(n t)}} I\{\tau(n)>n t(1+\varepsilon)\} .
\end{aligned}
$$

Thus, for any $\varepsilon_{1} \in(0,1)$

$$
\begin{aligned}
& \mathbf{P}\left(1-f_{\tau(n t)+R, N}(0) \geq \varepsilon_{1} ; \mathcal{H}_{n}(\varepsilon) ; \tau(n) \geq n t\right) \\
& \quad \leq \varepsilon_{1}^{-1} \mathbf{E}\left[1-f_{\tau(n t)+R, N}(0) ; \mathcal{H}_{n}(\varepsilon) ; \tau(n) \geq n t\right] \\
& \quad \leq \varepsilon_{1}^{-1} \mathbf{E}\left[e^{S_{\tau(n)}-S_{\tau(n t)}} ; \tau(n)>n t(1+\varepsilon)\right] .
\end{aligned}
$$

Hence, using the notation introduced in the proof of Lemma 10 and the duality principle for random walks it is not difficult to check that

$$
\begin{aligned}
\mathbf{E} & {\left[e^{S_{\tau(n)}-S_{\tau(n t)}} ; \tau(n)>n t(1+\varepsilon)\right] } \\
& =\sum_{n t(1+\varepsilon)<k \leq n} \mathbf{E}\left[e^{S_{k}-\min _{0 \leq i \leq n t} S_{i}} ; \tau(n)=k\right] \\
& =\sum_{n t(1+\varepsilon)<k \leq n} \mathbf{E}\left[e^{\min _{k-n t \leq l \leq k} S_{l}^{-}} ; A_{k, n-k}\right] \\
& \leq \sum_{n t(1+\varepsilon)<k \leq n} \mathbf{E}\left[e^{\min _{n t \varepsilon \leq l \leq k} S_{l}^{-}} \mid A_{k, n-k}\right] \mathbf{P}(\tau(n)=k) .
\end{aligned}
$$

Since $S_{l}^{-} \rightarrow-\infty \hat{\mathbf{P}}$-a.s as $l \rightarrow \infty$, we have by Lemma 4

$$
\lim _{n \rightarrow \infty} \mathbf{E}\left[e^{\min _{n t \varepsilon \leq l \leq k} S_{l}^{-}} \mid A_{k, n-k}\right]=0 .
$$


Now the dominated convergence theorem gives

$$
\lim _{n \rightarrow \infty} \mathbf{E}\left[e^{S_{\tau(n)}-S_{\tau(n t)}} ; \tau(n)>n t(1+\varepsilon)\right]=0 .
$$

Combining this fact with (31) complete the proof of the lemma.

Lemma 16 Assume that $A 1-A 2$ hold. For any $R \in \mathbb{Z}$ and any $t \in(0,1)$

$$
\left\{\frac{1-f_{0, \tau(n t)+R}(0)}{e^{S_{\tau(n t)+R}}} \mid \tau(n) \geq n t\right\} \stackrel{d}{\rightarrow} \Theta_{R}
$$

where $\Theta_{R}$ is the same as in Theorem 1

Proof. Let $R \geq 0$ be fixed. We have

$$
\begin{aligned}
& \mathbf{E} {\left[\exp \left\{-\lambda \frac{1-f_{0, \tau(n t)+R}(0)}{e^{S_{\tau(n t)+R}}}\right\} ; \tau(n) \geq n t\right] } \\
&= \mathbf{E}\left[\operatorname { e x p } \left\{-\lambda \frac{1-f_{0, \tau(n t)+R}(0)}{\left.\left.e^{S_{\tau(n t)+R}}\right\}\right]}\right.\right. \\
&-\mathbf{E}\left[\exp \left\{-\lambda \frac{1-f_{0, \tau(n t)+R}(0)}{e^{S_{\tau(n t)+R}}}\right\} ; \tau(n)<n t\right] .
\end{aligned}
$$

Introducing once again two independent environmental sequences $\left\{f_{n}^{-}, n \geq 0\right\}$ and $\left\{f_{n}^{+}, n \geq 0\right\}$, setting

$$
T_{\tau(n t), n t}^{*}:=\exp \left\{-\lambda \frac{1-f_{0, \tau(n t)+R}(0)}{e^{S_{\tau(n t)+R}}}\right\}, T_{l, n t-l}:=\exp \left\{-\lambda \frac{1-f_{l, 0}^{-}\left(f_{0, R}^{+}(0)\right)}{e^{S_{l}^{-}} e^{S_{R}^{+}}}\right\}
$$

and observing that

$$
\frac{1-f_{l, 0}^{-}\left(f_{0, R}^{+}(0)\right)}{e^{S_{l}^{-}} e^{S_{R}^{+}}} \rightarrow \frac{\zeta^{-}\left(f_{0, R}^{+}(0)\right)}{e^{S_{R}^{+}}}
$$

$\hat{\mathbf{P}}$ a.s. as $\min (l, n(1-t)-l) \rightarrow \infty$, we conclude by Lemma 5 that for any $\lambda \in(0, \infty)$

$$
\mathbf{E}\left[\exp \left\{-\lambda \frac{1-f_{0, \tau(n t)+R}(0)}{e^{S_{\tau(n t)+R}}}\right\}\right] \rightarrow \hat{\mathbf{E}}\left[\exp \left\{-\lambda \frac{\zeta^{-}\left(f_{0, R}^{+}(0)\right)}{e^{S_{R}^{+}}}\right\}\right]
$$

as $n \rightarrow \infty$. Further, the same as in Lemma 15

$$
\begin{aligned}
& \mathbf{E}\left[\operatorname { e x p } \left\{-\lambda \frac{1-f_{0, \tau(n t)+R}(0)}{\left.\left.e^{S_{\tau(n t)+R}}\right\} ; \tau(n)<n t\right]}\right.\right. \\
& =\mathbf{E}\left[\operatorname { e x p } \left\{-\lambda \frac{1-f_{0, \tau(n)+R}(0)}{\left.\left.e^{S_{\tau(n)+R}}\right\} ; \tau(n)<n t\right]}\right.\right. \\
& =\sum_{j=0}^{n t-1} \mathbf{E}\left[\exp \left\{-\lambda \frac{1-f_{0, j+R}(0)}{e^{S_{j+R}}}\right\} ; \tau(n)=j\right]
\end{aligned}
$$




$$
\begin{aligned}
& =\sum_{j=0}^{n t-1} \mathbf{E}\left[\exp \left\{-\lambda \frac{1-f_{0, j+R}(0)}{e^{S_{j+R}}}\right\} ; \min _{0 \leq r<j} S_{r}>S_{j}, \min _{j+1 \leq r \leq n} S_{r} \geq S_{j}\right] \\
& =\sum_{j=0}^{n t-1} \mathbf{E}\left[\exp \left\{-\lambda \frac{1-f_{j, 0}^{-}\left(f_{0, R}^{+}(0)\right)}{e^{S_{j}^{-}} e^{S_{R}^{+}}}\right\} ; A_{j, n-j}\right] \\
& =\sum_{j=0}^{n t-1} \mathbf{E}\left[\exp \left\{-\lambda \frac{1-f_{j, 0}^{-}\left(f_{0, R}^{+}(0)\right)}{e^{S_{j}^{-}} e^{S_{R}^{+}}}\right\} \mid A_{j, n-j}\right] \mathbf{P}(\tau(n)=j) .
\end{aligned}
$$

By (14)

$$
\frac{1-f_{j, 0}^{-}\left(f_{0, R}^{+}(0)\right)}{e^{S_{j}^{-}} e^{S_{R}^{+}}} \rightarrow \frac{\zeta^{-}\left(f_{0, R}^{+}(0)\right)}{e^{S_{R}^{+}}}
$$

$\hat{\mathbf{P}}$ a.s. as $\min (j, n-j) \rightarrow \infty$. Hence we see by Lemma 4 that

$$
\lim _{\min (j, n-j) \rightarrow \infty} \mathbf{E}\left[\exp \left\{-\lambda \frac{\zeta^{-}\left(f_{0, R}^{+}(0)\right)}{e^{S_{R}^{+}}}\right\} \mid A_{j, n-j}\right]=\hat{\mathbf{E}}\left[\exp \left\{-\lambda \frac{\zeta^{-}\left(f_{0, R}^{+}(0)\right)}{e^{S_{R}^{+}}}\right\}\right] .
$$

This and the generalized arcsine law give

$$
\begin{gathered}
\lim _{n \rightarrow \infty} \mathbf{E}\left[\exp \left\{-\lambda \frac{1-f_{0, \tau(n t)+R}(0)}{e^{S_{\tau(n t)+R}}}\right\} ; \tau(n)<n t\right] \\
=\hat{\mathbf{E}}\left[\exp \left\{-\lambda \frac{\zeta^{-}\left(f_{0, R}^{+}(0)\right)}{e^{S_{R}^{+}}}\right\}\right] \mathbf{P}(\tau \leq t) .
\end{gathered}
$$

Thus,

$$
\begin{gathered}
\lim _{n \rightarrow \infty} \mathbf{E}\left[\exp \left\{-\lambda \frac{1-f_{0, \tau(n t)+R}(0)}{e^{S_{\tau(n t)+R}}}\right\} ; \tau(n) \geq n t\right] \\
=\hat{\mathbf{E}}\left[\exp \left\{-\lambda \frac{\zeta^{-}\left(f_{0, R}^{+}(0)\right)}{e^{S_{R}^{+}}}\right\}\right] \mathbf{P}(\tau \geq t)
\end{gathered}
$$

and the statement of the lemma for $R \geq 0$ follows. The case $R<0$ can be treated in a similar way.

Lemma 17 Assume that $A 1-A 2$ hold. For any $R \in \mathbb{Z}$, any $t \in(0,1)$ and $N \in\{n-1, n\}$

$$
\left\{O_{\tau(n t)+R, N} \mid \tau(n) \geq n t\right\} \stackrel{d}{\rightarrow} \frac{1}{\Theta_{R}}-1 .
$$

Proof. We have

$$
O_{\tau(n t)+R, N}=f_{\tau(n t)+R, N}(0) \beta_{N}(\tau(n t)+R)\left(\frac{e^{S_{\tau(n t)+R}}}{1-f_{0, \tau(n t)+R}(0)}-1\right) .
$$


Applying Lemmas 14, 15, and 16 we get

$$
\left\{O_{\tau(n t)+R, N} \mid \tau(n) \geq n t\right\} \stackrel{d}{\rightarrow} \frac{1}{\Theta_{R}}-1
$$

as desired.

Lemma 18 Assume that $A 1-A 2$ hold. Then

$$
\Delta_{m, n} \stackrel{p}{\rightarrow} 1 \text { as } \min (m, n-m) \rightarrow \infty .
$$

Proof. Clearly, it suffices to show that

$$
\frac{1-f_{0, n}(0)}{1-f_{0, n-1}(0)} \stackrel{p}{\rightarrow} 1 \text { as } n \rightarrow \infty .
$$

To verify this write

$$
T_{\tau(n-1), n-1}^{*}:=\frac{1-f_{0, \tau(n-1)}\left(f_{\tau(n-1), n}(0)\right)}{1-f_{0, \tau(n-1)}\left(f_{\tau(n-1), n-1}(0)\right)}
$$

and

$$
T_{l, n-l-1}:=\frac{1-f_{l, 0}^{-}\left(f_{0, n-l-1}^{+}\left(f_{n-1}(0)\right)\right)}{1-f_{l, 0}^{-}\left(f_{0, n-l-1}^{+}(0)\right)} .
$$

Since, evidently, there exists $s \in[0,1)$ such that

$$
\lim _{n-l \rightarrow \infty} f_{n-l-1}^{+}(s) \neq s,
$$

it follows from Lemma 1' and Theorem 5 in (3) and 11 that $\hat{\mathbf{P}}^{+}$a.s.

$$
\lim _{n-l \rightarrow \infty} f_{0, n-l-1}^{+}\left(f_{n-1}(0)\right)=\lim _{n-l \rightarrow \infty} f_{0, n-l-1}^{+}(0)=q^{+} .
$$

Hence we conclude that $\hat{\mathbf{P}}$ a.s.

$$
\begin{aligned}
& \lim _{\min (l . n-l) \rightarrow \infty} T_{l, n-l-1} \\
= & \lim _{\min (l . n-l) \rightarrow \infty} \frac{1-f_{l, 0}^{-}\left(f_{0, n-l-1}^{+}\left(f_{n-1}(0)\right)\right)}{e^{S_{l}^{-}}} \times \frac{e^{S_{l}^{-}}}{1-f_{l, 0}^{-}\left(f_{0, n-l-1}^{+}(0)\right)}=1 .
\end{aligned}
$$

To finish the proof of 32 it remains to observe that

$$
\lim _{n \rightarrow \infty} \mathbf{P}(\tau(n) \neq \tau(n-1))=0,
$$

to check that

$$
\mathbf{E}\left[T_{\tau(n-1), n-1}^{*} \mid \tau(n-1)=l\right]=\mathbf{E}\left[T_{l, n-l-1} \mid A_{l, n-l-1}\right],
$$

and to apply Lemma 5 .

Proof of Theorem 1 To prove the statement of the theorem it suffices to combine Lemmas 17, 13, and 18. 


\section{Proof of Theorem 2}

Lemma 19 Under Assumptions $A 1-A 2$,

$$
\frac{O_{n t, n-1}}{O_{n t, n}} \stackrel{p}{\rightarrow} 1 \text { as } n \rightarrow \infty
$$

Proof. We have

$$
\frac{O_{n t, n-1}}{O_{n t, n}}=\frac{f_{n t, n-1}(0)}{f_{n t, n}(0)} \times \frac{1-f_{0, n-1}(0)}{1-f_{0, n}(0)} \times \frac{1-f_{n t, n}(0)}{1-f_{n t, n-1}(0)} .
$$

Since the process is critical,

$$
f_{n t, n}(0) \stackrel{d}{=} f_{0, n(1-t)}(0) \rightarrow 1 \text { a.s. }
$$

as $n \rightarrow \infty$. Similarly to 32

$$
\frac{1-f_{n t, n}(0)}{1-f_{n t, n-1}(0)} \stackrel{d}{=} \frac{1-f_{0, n(1-t)}(0)}{1-f_{0, n(1-t)-1}(0)} \stackrel{d}{\rightarrow} 1
$$

as $n \rightarrow \infty$. Combining (32), (34), and 35) gives 33).

Lemma 20 Under Assumptions $A 1-A 2$, for any $t \in(0,1)$ as $n \rightarrow \infty$

$$
\left\{O_{n t, n}\left(1-f_{0, n t}(0)\right) e^{-S_{n t}} \mid \tau(n) \geq n t\right\} \stackrel{d}{\rightarrow} 1 .
$$

and

$$
\left\{O_{n t, n}\left(1-f_{n t, n}(0)\right) \mid \tau(n)<n t\right\} \stackrel{d}{\rightarrow} 1,
$$

Proof. To prove 36 , one should write

$$
\left.O_{n t, n}\left(1-f_{0, n t}(0)\right) e^{-S_{n t}}=f_{n t, n}(0) \beta_{n}(n t)\left[1-\left(1-f_{n t, n}(0)\right)\right) e^{-S_{n t}}\right]
$$

and to use Lemma 12 and 25 , while to demonstrate 37 it suffices to observe that

$$
O_{n t, n}\left(1-f_{n t, n}(0)\right)=\frac{1}{\alpha_{n}(n t)} \times f_{n t, n}(0)-f_{n t, n}(0) \beta_{n}(n t)\left(1-f_{n t, n}(0)\right)
$$

and to apply Lemma 8 and 26 .

Lemma 21 Under Assumptions $A 1-A 2$, for any $t \in(0,1)$ as $n \rightarrow \infty$

$$
\left\{\frac{1-f_{0, n t}(0)}{e^{S_{\tau(n t)}}} \mid \tau(n) \geq n t\right\} \rightarrow \zeta
$$

and

where $\zeta$ is defined in (14).

$$
\left\{\frac{1-f_{n t, n}(0)}{e^{S_{\tau(n t, n)}-S_{n t}}} \mid \tau(n)<n t\right\} \rightarrow \zeta
$$


Proof. Our arguments follow the same line of reasoning as the proof of Lemma 16 We have

$$
\begin{aligned}
\mathbf{E}\left[\exp \left\{-\lambda \frac{1-f_{0, n t}(0)}{e^{S_{\tau(n t)}}}\right\} ; \tau(n) \geq n t\right] \\
=\mathbf{E}\left[\exp \left\{-\lambda \frac{1-f_{0, n t}(0)}{e^{S_{\tau(n t)}}}\right\}\right] \\
\quad-\mathbf{E}\left[\exp \left\{-\lambda \frac{1-f_{0, n t}(0)}{e^{S_{\tau(n t)}}}\right\} ; \tau(n)<n t\right] .
\end{aligned}
$$

Introducing once again two independent environmental sequences $\left\{f_{n}^{-}, n \geq 0\right\}$ and $\left\{f_{n}^{+}, n \geq 0\right\}$, setting

$$
T_{\tau(n t), n t}^{*}:=\exp \left\{-\lambda \frac{1-f_{0, n t}(0)}{e^{S_{\tau(n t)}}}\right\}, T_{l, n t-l}:=\exp \left\{-\lambda \frac{1-f_{l, 0}^{-}\left(f_{0, n t-l}^{+}(0)\right)}{e^{S_{l}^{-}}}\right\}
$$

and observing that by 14

$$
\frac{1-f_{l, 0}^{-}\left(f_{0, n t-l}^{+}(0)\right)}{e^{S_{l}^{-}}} \rightarrow \zeta^{-}\left(q^{+}\right)=\zeta
$$

$\hat{\mathbf{P}}$ a.s. as $\min (l, n t-l) \rightarrow \infty$, we conclude by Lemma 5 that for any $\lambda \in(0, \infty)$

$$
\mathbf{E}\left[\exp \left\{-\lambda \frac{1-f_{0, n t}(0)}{e^{S_{\tau(n t)}}}\right\}\right] \rightarrow \hat{\mathbf{E}}[\exp \{-\lambda \zeta\}]
$$

as $n \rightarrow \infty$. Further,

$$
\begin{aligned}
\mathbf{E} & {\left[\operatorname { e x p } \left\{-\lambda \frac{1-f_{0, n t}(0)}{\left.\left.e^{S_{\tau(n t)}}\right\} ; \tau(n)<n t\right]}\right.\right.} \\
& =\mathbf{E}\left[\operatorname { e x p } \left\{-\lambda \frac{1-f_{0, n t}(0)}{\left.\left.e^{S_{\tau(n)}}\right\} ; \tau(n)<n t\right]}\right.\right. \\
& =\sum_{j=0}^{n t-1} \mathbf{E}\left[\exp \left\{-\lambda \frac{1-f_{0, j}\left(f_{j, n t}(0)\right)}{e^{S_{j}}}\right\} ; \tau(n)=j\right] \\
& =\sum_{j=0}^{n t-1} \mathbf{E}\left[\exp \left\{-\lambda \frac{1-f_{0, j}\left(f_{j, n t}(0)\right)}{e^{S_{j}}}\right\} ; \min _{0 \leq r<j} S_{r}>S_{j}, \min _{j+1 \leq r \leq n} S_{r} \geq S_{j}\right] \\
& =\sum_{j=0}^{n t-1} \mathbf{E}\left[\exp \left\{-\lambda \frac{1-f_{j, 0}^{-}\left(f_{0, n t-j}^{+}(0)\right)}{e^{S_{j}^{-}}}\right\} ; A_{j, n-j}\right] \\
& =\sum_{j=0}^{n t-1} \mathbf{E}\left[\exp \left\{-\lambda \frac{1-f_{j, 0}^{-}\left(f_{0, n t-j}^{+}(0)\right)}{e^{S_{j}^{-}}}\right\} \mid A_{j, n-j}\right] \mathbf{P}(\tau(n)=j) .
\end{aligned}
$$

By (14)

$$
\frac{1-f_{j, 0}^{-}\left(f_{0, n t-j}^{+}(0)\right)}{e^{S_{j}^{-}}} \rightarrow \zeta
$$


$\hat{\mathbf{P}}$ a.s. as $\min (j, n t-j) \rightarrow \infty$. Hence we see by Lemma 4 that

$$
\lim _{\min (j, n t-j) \rightarrow \infty} \mathbf{E}\left[\exp \left\{-\lambda \frac{1-f_{j, 0}^{-}\left(f_{0, n t-j}^{+}(0)\right)}{e^{S_{j}^{-}}}\right\} \mid A_{j, n-j}\right]=\hat{\mathbf{E}}[\exp \{-\lambda \zeta\}] .
$$

This and the generalized arcsine law give

$$
\begin{aligned}
& \lim _{n \rightarrow \infty} \mathbf{E}\left[\exp \left\{-\lambda \frac{1-f_{j, 0}^{-}\left(f_{0, n t-j}^{+}(0)\right)}{e^{S_{j}^{-}}}\right\} ; \tau(n)<n t\right] \\
& =\hat{\mathbf{E}}[\exp \{-\lambda \zeta\}] \mathbf{P}(\tau \leq t) .
\end{aligned}
$$

Thus,

$$
\begin{aligned}
& \lim _{n \rightarrow \infty} \mathbf{E}\left[\exp \left\{-\lambda \frac{1-f_{0, n t}(0)}{e^{S_{\tau(n t)}}}\right\} ; \tau(n) \geq n t\right] \\
& =\mathbf{\mathbf { E }}[\exp \{-\lambda \zeta\}] \mathbf{P}(\tau \geq t)
\end{aligned}
$$

and the first statement of the lemma follows. The second statement can be checked in a similar way.

Lemma 22 Under Assumptions $A 1-A 2$, for any $t \in(0,1)$

$$
O_{n t, n} \stackrel{d}{\rightarrow} \infty \text { as } n \rightarrow \infty
$$

Proof. This statement is a direct corollary of Lemmas 20 and 21

Proof of Theorem 2. Using Lemma 3 we have

$$
\begin{aligned}
\frac{1}{1+O_{n t, n-1}\left(1-e^{-\lambda / O_{n t, n-1}}\right)} \Delta_{n t, n}^{-1} & \leq \mathbf{E}_{\overline{\mathbf{K}}}\left[\exp \left\{-\lambda \frac{Z_{n t}}{O_{n t, n}}\right\} \mid T=n\right] \\
& \leq \frac{1}{1+O_{n t, n}\left(1-e^{-\lambda / O_{n t, n}}\right)} \Delta_{n t, n} .
\end{aligned}
$$

Now to complete the proof of the theorem it remains to observe that

$$
\lim _{n \rightarrow \infty} \Delta_{n t, n} \stackrel{p}{=} 1
$$

by Lemmas 19 and 22 and 35 , and that by Lemma 22

$$
\lim _{n \rightarrow \infty} O_{n t, n}\left(1-e^{-\lambda / O_{n t, n}}\right) \stackrel{d}{=} \lim _{n \rightarrow \infty} O_{n t, n-1}\left(1-e^{-\lambda / O_{n t, n}}\right) \stackrel{d}{=} \lambda .
$$

Remark 23 It follows from Lemmas 20 and 21 that, as $n \rightarrow \infty$

$$
\left\{\frac{O_{n t, n}}{e^{S_{n t}-S_{\tau(n t)}}} \mid \tau(n) \geq n t\right\} \rightarrow \zeta
$$


and

$$
\left\{\frac{O_{n t, n}}{e^{S_{n t}-S_{\tau(n t, n)}}} \mid \tau(n)<n t\right\} \rightarrow \zeta .
$$

Thus, given $\{T=n\}$ the growth rate of $Z_{n t}$ depends essentially on the location of the point $\tau(n)$ of the global maximum of $\left\{S_{k}, 0 \leq k \leq n\right\}$ with respect to the moment $n t$. If $\tau(n) \geq n t$ then this growth rate is specified by the past local minimum of the associated random walk while if $\tau(n)<n t$ then it depends on the value of this random walk at the point of prospective minimum of its remaining piece.

\section{Acknowledgements}

Part of this work was carried out whilst V.A.V. was visiting A.E.K. at The Department of Mathematical Sciences at Heriot Watt University during the months of June and July 2006. V.A.V. is grateful for the department's hospitality during this period.

The authors are also thankful to the referees for valuable remarks allowing to improve the presentation.

\section{References}

[1] Afanasyev V.I., Geiger J., Kersting G., Vatutin V.A. Criticality for branching processes in random environment.- Ann. Probab., 33(2005), p. 645-673.

[2] Athreya K.B. and Karlin S. Branching processes with random environments, I: Extinction probability. - Ann. Math. Stat., 42(1971), p. 1499-1520.

[3] Athreya K.B. and Karlin S. On branching processes with random environments, II: limit theorems.Ann. Math. Statist., 42(1971), p. 1843-1858.

[4] Athreya K.B. and Ney P.E. Branching Processes. Springer-Verlag, Berlin, 1972.

[5] Bertoin, J. and Doney, R. A. On conditioning a random walk to stay nonnegative. - Ann. Probab., 22 (1994), p. 2152-2167.

[6] Borovkov K.A., Vatutin V.A. Reduced critical branching processes in random environment.- Stoch. Proc. Appl., 71 (1997), p. 225-240.

[7] Doney R.A. Spitzer's condition and the ladder variables in random walks. - Probab. Theory Relat. Fields , 101(1995), p. 577-580.

[8] Feller W. An Introduction to Probability Theory and its Applications. V.2, Willey, New York London-Sydney-Toronto, 1971.

[9] Fleischmann K., Vatutin V.A. Reduced subcritical branching processes in random environment.Adv. Appl. Probab., 31(1999), p. 88-111.

[10] Geiger J., Kersting G. The survival probability of a critical branching process in random environment.- Theory Probab. Appl., 45 (2000), p. 607-615.

[11] Spitzer F. Principles of random walk. Princeton NJ, Toronto - New York - London, 1964. 
[12] Vatutin V.A. Critical Reduced processes in random environment.- Theory Probab. Appl., 47(2003), p. 99-113.

[13] Vatutin V.A., Dyakonova E.E. Reduced branching processes in random environment. - In: Mathematics and Computer Science II: Algorithms, Trees, Combinatorics and Probabilities (Ed. B.Chauvin, P.Flajolet, D.Gardy, A.Mokkadem), Basel - Boston- Berlin: Birkhäuser, 2002, p. 455467.

[14] Vatutin V.A., Dyakonova E.E. Spitzer's condition and branching processes in random environment. - In: Mathematics and Computer Science III: Algorithms, Trees, Combinatorics and Probabilities (Ed. M.Drmota, P.Flajolet, D.Gardy, B.Gittenberger), Basel - Boston- Berlin: Birkhäuser, 2004, p. 375-385.

[15] Vatutin V.A. and Dyakonova E.E. Galton-Watson branching processes in random environment, I: limit theorems. - Theory Probab. Appl., 48(2003), p. 314-336.

[16] Vatutin V.A. and Dyakonova E.E. Galton-Watson branching processes in random environment, II: joint distributions. Theory Probab. Appl., 49(2004), p. 275-308.

[17] Vatutin V.A. and Dyakonova E.E. Branching processes in random environment and bottlenecks in the evolution of populations. Theory Probab. Appl., 51(2006), p. 189-210.

[18] Vatutin V.A. and Dyakonova E.E. Reduced branching processes in random environment. Theory Probab. Appl., 52(2007), p. 271-300 (In Russain).

[19] Vatutin V.A., Dyakonova E.E. Waves in reduced branching processes in random environment. Theory Probab. Appl., 53(2008), in print. 
